Rapid Reviews COVID-19

\title{
Review 4: "Neutralization of N501Y mutant SARS- CoV-2 by BNT162b2 vaccine-elicited sera"
}

Binquan Luan', Haoran Wang ${ }^{2}$

${ }^{1}$ IBM T J Watson Research, Computational Biological Center, USA, ${ }^{2}$ Neoland Bioscience

Published on: Feb 07, 2021

DOI: $10.1162 / 2 e 3983 f 5 . a 04 d 4 d 64$

License: Creative Commons Attribution 4.0 International License (CC-BY 4.0). 


\section{$\underline{\text { RR:C19 Evidence Scale rating by reviewer: }}$}

- Strong. The main study claims are very well-justified by the data and analytic methods used. There is little room for doubt that the study produced has very similar results and conclusions as compared with the hypothetical ideal study. The study's main claims should be considered conclusive and actionable without reservation.

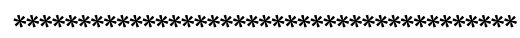

\section{Review:}

The spike protein of SARS-CoV-2 is a key target of virus-neutralizing antibodies, hence any mutation that occurs in the corresponding sequence naturally raises significant concerns about the efficacy of existing COVID-19 vaccines, especially those using it as the antigen. In this study, the authors performed in vitro experiment to evaluate the efficacy of the mRNA-based COVID-19 vaccine, examining whether the N501Y mutation could cause the virus to undermine the vaccine. With their generated isogenic N501 and Y501 SARS-CoV-2 and sera of 20 participants in a previously reported trial of the vaccine BNT162b2, they found that the sera had equivalent neutralizing titers to both the N501 and Y501 viruses. We found that this is an important and first-hand study to assess the vaccine's effectiveness against new SARS-CoV-2 variants. Also, it is a timely study and we strongly support its publication. However, we hope that the authors can clarify the importance of their study to avoid any possible misunderstanding.

The authors provided important insights into the neutralizing capability of sera after the BNT162b2 vaccination towards the prevailing SARS-CoV-2 N501Y mutation found in recent UK and South Africa variants. Based on their experiments, SARS-CoV-2 N501Y isogenic variant indeed exhibited no obvious resistance to clinical sera. As we pointed out previously (see https://www.biorxiv.org/content/10.1101/2021.01.04.425316v1), the N501Y variant may have formed better hydrophobic interactions with ACE2 albeit there is no clear indication that this mutation facilitates the virus to evade neutralizing antibodies more easily. In this respect, we found that their results are encouraging and confirm that the vaccine will likely be effective against the UK strain.

However, we must be warned that the South Africa strain 501Y.V2 has two more mutations (K417N and $\mathrm{E} 484 \mathrm{~K}$ ) other than N501Y on the receptor-binding domain (RBD) of the spike protein. Recent studies show that these two mutations indeed can help the variant to evade neutralizing antibodies (see https://www.biorxiv.org/content/10.1101/2021.01.18.427166v1). Therefore, it is premature to state that the vaccine is also effective for the 501Y.V2 strain and further experiments are required. We hope that the authors can clarify this important point and avoid any possible misunderstanding. 
Further, mutations such as K417N and E484K in combination with N501Y probably conferred the balance between maintained ACE2 binding and much-elevated antibody evasion properties. In the in vitro experiment, it is also important to know how much these mutations have increased their bindings with the human ACE2 receptor, compared with their bindings with neutralizing human antibodies.

In summary, we support the publication of this work in a timely fashion. Nonetheless, it is also highly recommended that the authors should extend their experimental study of the vaccine for the South Africa strain (501Y.V2) as soon as possible.

\section{Disclaimer:}

The authors declare that B.L is an employee of IBM and H.W. is an employee of Neoland Bioscience. The employers were not involved in the analysis/interpretation of data and the writing of this article. 